\title{
PARAMETERIZATION OF MODEL VALIDATING SETS FOR UNCERTAINTY BOUND OPTIMIZATIONS *
}

\author{
K. B. Lim L D. P. Giesy $^{\ddagger}$ \\ NASA Langley Research Center \\ Hampton, Virginia
}

\begin{abstract}
Given measurement data, a nominal model and a linear fractional transformation uncertainty structure with an allowance on unknown but bounded exogenous disturbances, easily computable tests for the existence of a model validating uncertainty set are given. Under mild conditions, these tests are necessary and sufficient for the case of complex, nonrepeated, block-diagonal structure. For the more general case which includes repeated and/or real scalar uncertainties, the tests are only necessary but become sufficient if a collinearity condition is also satisfied. With the satisfaction of these tests, it is shown that a parameterization of all model validating sets of plant models is possible. The new parameterization is used as a basis for a systematic way to construct or perform uncertainty tradeoff with model validating uncertainty sets which have specific linear fractional transformation structure for use in robust control design and analysis. An illustrative example which includes a comparison of candidate model validating sets is given.
\end{abstract}

\section{Introduction}

In applying multivariable robust control analysis and synthesis techniques to linear, timeinvariant systems, a particular set of plant models described by a nominal model, uncertainty

\footnotetext{
${ }^{*}$ Earlier version presented at the AIAA Guidance, Navigation and Control Conference, Boston, MA, August 10-12, 1998, as paper No. 98-4135.

${ }^{\dagger}$ Aerospace Technologist, Guidance \& Control Branch, k.b.lim@larc.nasa.gov

${ }_{\ddagger}^{\ddagger}$ Mathematician, Guidance \& Control Branch, d.p.giesy@larc.nasa.gov
} 
structure, and norm bounds on the model uncertainty and exogenous inputs is required a priori $[1,2]$. In a typical setting, a controls engineer selects or develops a best possible model either from first principles and/or from system identification or parameter estimation. In situations where the physical conditions are not accurately known nor reliable, the engineer selects a model uncertainty structure around a best possible nominal model in order to represent a set of plant models so that robustness of the system can be analyzed and optimized through feedback control. In many instances where measurements are available, the engineer can check to see if the given set of plants is consistent with the available measurement data. Specifically [3]-[4], given a robust control design model, does a plant exist within this set which will reproduce a given input and output measurements, i.e., is the given set of plants model validating (or more accurately cannot be invalidated) with respect to available data?

There exists a wealth of literature related to model validation, uncertainty modeling and identification for control (see, for example [5]). Although there have been several successful methodology validation tests reported recently as in [6]-[10], it appears that there is not a systematic methodology that can be demonstrated to work for a significant class of problems. Indeed, this study addresses a variant model validation problem originally formulated in [3]. In [3], a smallest level of exogenous disturbance that satisfies model validation given a fixed level of model uncertainty bound is sought whereas in this paper, we seek a smallest model uncertainty bound that satisfies model validation given an allowance on the exogenous disturbance. Furthermore, exogenous noise signals of the type $B \ell^{(2)}$ is assumed in [3] while we assume that the exogenous noise is a colored white noise signal whose power spectrum is a known constant over a wideband frequency, i.e. belongs to $B \ell^{(\infty)}$.

The model validation question has a binary outcome which can be viewed as a check on two properties. First, the postulated parameteric and nonparametric uncertainties and their corresponding interconnections leading to a Linear Fractional Transformation (LFT) structure is required to be sufficiently rich to admit a perturbed model that will faithfully reproduce the measured input/output data. In the seminal work in [3], this property is 
referred to as a feasible set. Second, assuming feasibility, the postulated norm bound on the uncertainties must be sufficiently large to admit a model validating plant. The viewpoint taken in this paper with regards to model validation is to focus on the first property, namely whether an a priori given LFT structure for an uncertainty model can lead to a model validating set given some allowance in exogenous disturbance. The rationales for the above viewpoint are: (i) feasibility question is simpler to address, (ii) if feasibility is not satisfied, there is no point in checking the second property, (iii) once feasibility is satisfied, a model validating set can almost always be constructed, using the results in this paper. The above viewpoint suggests a slight adjustment in the model validation paradigm, namely, it is not of prime significance whether a particular set satisfies model validation since if feasibility is satisfied, model validating sets are highly non-unique.

For brevity, we highlight only closely related earlier work to our approach. In an early attempt to obtain a simpler problem formulation and solution than the approach taken by [3] to [6], [8] considered a special case where all exogenous inputs are either known or are very small and occur only at the output. This attempt was subsequently applied to an experimental testbed with encouraging results [9]. Although the approach taken in [8], [9] appears to work reasonably well for problems with an arbitrary number of structured full complex blocks only, it became clear through applications that problems with parametric (and often real repeated) uncertainties gave unsatisfactory results. This was somewhat expected because the additional structure in the repeated scalar uncertainties was not incorporated in the original problem formulation. Hence, the most recent work reported in [11] extends the previous approach to include repeated and/or real scalar parametric uncertainties along with an arbitrary number of full complex blocks. This paper provides the detailed proofs omitted in [11], expands the uncertainty set to include unknown but bounded output noise and/or disturbance to the plant, and ultimately parameterize all model validating sets for a fixed LFT structure. We also discuss and provide a means to cope with the issue of determining non-parametric uncertainty bounds in the presence of constant parametric uncertainties. 
The formulation in this paper is for a closed loop system and the open loop configuration is obtained as a special case.

In Section 2, a problem definition is given whereby uncertainty bounds are viewed as bounds on fictitious uncertainty signals which satisfy $P-\Delta$ transmission conditions while resulting in zero output errors. In Section 3 we derive existence conditions for a model validating set followed by a parameterization of all model validating sets and associated signals. In Section 4, we outline possibilities in utilizing the parameterizations given in Section 3. A smallest set formulation is discussed in which allowances are given to parametric uncertainties similar to the treatment of bounded but unknown exogenous random disturbances. A smallest set refers to a smallest normed set of non-parametric uncertainties, i.e., unmodeled dynamics. An illustrative example is given in section 5 which includes the computation and comparison of three model validating sets. Section 6 concludes the paper.

\section{Problem Definition}

For a given approximately linear and time-invariant physical system, suppose a controller, $K$, is known to be internally stabilizing and we can measure its inputs, $u$, command inputs, $r$, and the outputs, $y$, as shown in Figure 1. The exogenous disturbances consist of measurement noise at the output and exogenous disturbances either through the control input channel or through a separate path. To accommodate this, a noise filter, $V$, and a disturbance filter imbedded in the augmented nominal plant, $P$ are assumed. Both filters are assumed driven by unknown but bounded independent random signals. The closed loop output of the plant model, $\tilde{y}$, is a sum of responses due to filtered noise, $\nu$, and disturbances, $\epsilon$, known input signal, $r$, all subject to feedback between a known controller, $K$, and a plant which belongs to a set defined by an upper LFT model,

$$
F_{u}(P, \Delta):=\left[P_{22}, P_{23}\right]+P_{21} \Delta\left(I-P_{11} \Delta\right)^{-1}\left[P_{12}, P_{13}\right]
$$

where the structured uncertainty is defined by $\Delta \in \mathcal{D}$, where

$$
\mathcal{D}:=\left\{\Delta \in \mathcal{C}^{m \times n}: \Delta=\operatorname{diag}\left(\delta_{1} I_{n_{1}}, \ldots, \delta_{r} I_{n_{r}}, \Delta_{r+1}, \ldots, \Delta_{\tau}\right), \delta_{i} \in \mathcal{F}_{i}, \Delta_{i} \in \mathcal{C}^{m_{i} \times n_{i}}\right\}
$$


and $\tau$ denotes the number of uncertainty blocks and $\mathcal{F}_{i}$ is either the field $\mathcal{R}$ of real numbers or the field $\mathcal{C}$ of complex numbers at the designer's choosing. A corresponding set of bounded structured uncertainty is defined by

$$
\mathcal{D}_{W}:=\left\{\Delta \in \mathcal{D}: \Delta=\Delta_{B} W, \bar{\sigma}\left(\Delta_{B}\right) \leq 1\right\}
$$

where the elements of the diagonal matrix, $W:=\operatorname{diag}\left(w_{1} I_{n_{1}}, \ldots, w_{\tau} I_{n_{\tau}}\right)$, denotes the scaling radii applied to the blocks of the structured uncertainty unit ball as defined by $\bar{\sigma}\left(\Delta_{B}\right) \leq 1$.

For convenience, combine both exogenous disturbances $\epsilon$ and $\nu$ into a single disturbance vector

$$
\beta:=\left\{\begin{array}{c}
\epsilon \\
\nu
\end{array}\right\}
$$

The model in a canonical form is shown in Figure 1. For an open loop problem, $G(P, K, V)=$ $G(P, 0, V)$, and $r=u$, and all the remaining developments remain the same. Of course the coupling effects of the noise and disturbances with the model uncertainties are more complicated due to feedback. Note also that since $r$ and $K$ are assumed known, as is typical in many applications, if the output error, $e_{y}:=y-\tilde{y}=0$, then the input error, $e_{u}:=u-\tilde{u}=0$. This means that it is sufficient to impose $e_{y}=0$ only.

Suppose the measurements are taken in the discrete-time domain and consider a discrete frequency domain formulation, so called Constant Matrix case in [3]. For simplicity, we assume that a discrete Fourier transform has been performed and do not consider the additional affects of realistic signal conditioning operations typically performed on the raw discrete-time signals. Since $\bar{\sigma}(\Delta)=\max _{i} \bar{\sigma}\left(\Delta_{i}\right)$ and we are primarily concerned with the size of the uncertainty blocks, it is of interest to note that uncertainty bound in terms of its maximum singular value can be written as a ratio of norms

$$
\bar{\sigma}\left(\Delta_{i}\right):=\sup _{\eta_{i}^{\prime}} \frac{\left\|\Delta_{i} \eta_{i}^{\prime}\right\|}{\left\|\eta_{i}^{\prime}\right\|} \geq \frac{\left\|\xi_{i}\right\|}{\left\|\eta_{i}\right\|} \quad 1 \leq i \leq \tau
$$

where $\operatorname{col}\left(\xi_{1}, \cdots, \xi_{\tau}\right)$ and $\operatorname{col}\left(\eta_{1}, \cdots, \eta_{\tau}\right)$ are the partitioning of the vectors $\xi$ and $\eta$ which conform to the block diagonal partition of $\Delta$ in Equation (1). Of course fictitious signals $\xi$ and $\eta$ cannot be measured nor are they arbitrary so that it is necessary to look at their dependence 
on real signals $u$ and $y$ and their transmission through a postulated system $G(P, K, V)$ at each frequency. The signals, $\xi$ and $\eta$, whose norm ratios determine the uncertainty sizes, must be consistent with their transmission through the nominal plant model and uncertainty structure. The signals must also reproduce the measured inputs and outputs with some help from simulated exogenous disturbances which are assumed unknown but bounded by known bounds.

The output error is given by

$$
e_{y}:=y-\tilde{y}=e_{y}^{o}-G_{21} \xi-G_{22} \beta
$$

where $e_{y}^{o}:=y-G_{23} r$ denotes the nominal output error due to the nominal plant in closed loop. The terms $G_{21} \xi$ and $G_{22} \beta$ in Equation (5) represent the uncertainty freedoms in an attempt to negate the nominal output error. The first term corresponds to the uncertainty freedom in an LFT structured uncertainty model, and the second term is an allowance given to unknown but bounded exogenous noise and disturbances in the system.

\section{Definition (Model Validation) [3]:}

Given measurements of the input signal, $r$, output signal, $y$, and noise filter, $V$, an augmented nominal plant model, $P$, with a disturbance filter, a controller, $K$, and a set of bounded structured uncertainty, $\mathcal{D}_{W}$. The set of plants (robust control design models)

$$
\mathcal{P}_{W}:=\left\{F_{u}(P, \Delta), \Delta \in \mathcal{D}_{W}\right\}
$$

is said to be a model validating set if it contains an uncertainty model $\Delta \in \mathcal{D}_{W}$ such that there exists exogenous disturbance signals, $\epsilon, \nu$ with $\|\beta\| \leq 1$ for which

$$
y=\mathcal{F}_{u}(G(P, K, V), \Delta)\left\{\begin{array}{c}
\epsilon \\
\nu \\
r
\end{array}\right\} .
$$

In short, a set of plants is model validating if it can reproduce the given measurements while subject to a priori constraints, but of course, as noted earlier [3]-[6], one can never really "validate" a model since fresh data could potentially invalidate it. Notice that the assumption in the exogenous signal is different from the original problem definition in [3]. 
A comment is in order about the combining of the two exogenous disturbance vectors $\epsilon$ and $\nu$ into the single vector $\beta$. They are applied at dissimilar points of the plant model, and in many ways it would seem natural to keep them separate and, perhaps, define model validating set in terms of independent bounds on the two signals; typically, $\|\epsilon\| \leq 1$ and $\|\nu\| \leq 1$. However, the mathematics used in Lemma 2 in deriving the parameterization of model validating signals requires that $\beta$ be bounded in the $\ell^{(2)}$ (Euclidean) norm, and the argument would not work if, instead, $\beta$ were bounded by the hybrid $\ell^{(\infty)} / \ell^{(2)}$ norm which would be equivalent to the individual bounds on $\epsilon$ and $\nu$. However, the two conditions are fairly close to each other:

$$
\|\beta\| \leq 1 \Rightarrow\|\epsilon\| \leq 1 \text { and }\|\nu\| \leq 1 \Rightarrow\|\beta\| \leq \sqrt{2}
$$

In the case that one or the other of $\epsilon$ and $\nu$ is negligible, then $\|\beta\|$ is approximately equal to the norm of the other, and even in the general case, the discrepancy between the two bounding paradigms is only $\sqrt{2}$, independent of the dimensions of $\nu$ and $\epsilon$.

In this paper, we characterize model validation in a way that will allow a convenient parameterization of all model validating uncertainty sets with respect to available input/output measurement for a given LFT structure. The idea is that the engineer will ultimately select a particulat set of model validating uncertainty weights based on the particular application. To this end, we first investigate a feasibility condition (or necessary condition) for model validation: at each frequency, is there a pair $(\xi, \beta)$ subject to $\|\beta\| \leq 1$ which makes the output error in Equation (5) zero? Note that once $G, r, \beta$, and $\xi$ are specified, $\eta$ is completely determined. The next step is to incorporate the constraints due to a priori structure in the uncertainties which may limit the feasible $(\xi, \eta)$ signals. This leads to necessary and sufficient conditions for model validation. The final step is to parameterize all model validating sets of plant models.

It is clear that if the noise vector $\nu$ is not restricted by a fixed bound, then any output residual can be zeroed out (without any help from $\Delta$ and/or $\epsilon$ ) if the noise filter $V$ is non-singular. In this paper we assume that the noise filter $V$ is given (as part of the a 
priori model assumption or a reasonable model determined from earlier system identification experiments) and the noise vector at each frequency is norm bounded by 1 . This output noise model can be viewed as a model of a broad band exogenous noise typified by sensor noise. Of course when judicisouly chosen, $V$ can reflect a priori bounds on the noise intensity or power spectrum of the unknown exogenous signal over a bandwidth of interest. The input vector, $\epsilon$, is meant to denote unknown but bounded exogenous disturbances on the physical system. As an example, in aeroservoelasticity applications, $\epsilon$ will denote white noise input to a gust filter imbedded in $P_{12}$ and $P_{22}$.

\section{Parameterization of Model Validating Sets}

In this section, we develop a theory to effectively parameterize all model validating uncertainty sets which satisfy a priori assumptions on the LFT structure and exogenous disturbances. We begin by first addressing the question: when does there exist a norm bounded combined exogenous disturbance $\beta$ with $\|\beta\| \leq 1$ and a $\xi \in \mathcal{C}^{n_{\xi}}$ such that $e_{y}=0$ ? At this point, notice that $\xi$ is not required to be limited by any given bound or structure. To answer the above question we first state a lemma, set some notation, and make some observations.

\section{Lemma 1:}

Let $A$ be a matrix whose singular value decomposition (SVD) is

$$
A=\left[\begin{array}{ll}
U_{1} & U_{2}
\end{array}\right]\left[\begin{array}{cc}
\Sigma_{1} & 0 \\
0 & 0
\end{array}\right]\left[\begin{array}{l}
V_{1}^{H} \\
V_{2}^{H}
\end{array}\right]
$$

where $U=\left[\begin{array}{ll}U_{1} & U_{2}\end{array}\right]$ and $V=\left[V_{1} V_{2}\right]$ are unitary, $\Sigma_{1}$ is diagonal and nonsingular, and the block matrix partitionings are conformal. Let $b$ be a vector and $c$ a non-negative real constant. Then the inequality

$$
\|A x+b\| \leq c
$$

has a solution $x$ if and only if

$$
\left\|U_{2}^{H} b\right\| \leq c
$$


Then the general solution to the inequality (7) is parameterized by

$$
\begin{aligned}
& x=V_{1} y+V_{2} z \\
& y=\Sigma_{1}^{-1}\left(w-U_{1}^{H} b\right)
\end{aligned}
$$

where $z$ is arbitrary, and $w$ is any vector with

$$
\|w\| \leq \sqrt{c^{2}-\left\|U_{2}^{H} b\right\|^{2}}
$$

\section{Remark:}

One or both of two special circumstances may apply to the SVD of $A$ : $A$ might be of full row [column] rank in which case the $U_{2}\left[V_{2}\right]$ matrix is missing from the SVD, as is the bottom row [right column] of zeros in the diagonal matrix of singular values. The following modifications make Lemma 1 and its proof remain correct under any combination of these circumstances. If $A$ is of full row rank, then consider $\left\|U_{2}^{H} b\right\|$ to be 0 and remove the $U_{2}$ lines from Equations (12) and (13). If $A$ is of full column rank, then remove any reference to $z$ and $V_{2}$ and realize that $w$ subject to the inequality (11) is the only free parameter in the parameterization of solutions to the inequality (7). The necessary and sufficient condition in equation (8) is saying that the norm of the projection error of $b$ onto $U_{2}$ must be less than or equal to the given inequality bound.

\section{Proof of Lemma 1:}

Note that $x$ can always be written as $V_{1} y+V_{2} z$ by taking $y=V_{1}^{H} x$ and $z=V_{2}^{H} x$. Now, from the SVD of $A, A V_{2} z=0$, and $A V_{1} y=U_{1} \Sigma_{1} y$. Therefore, for arbitrary $y$ and $z$, if $x=V_{1} y+V_{2} z$ then $\|A x+b\|^{2} \leq c^{2}$ if and only if $\left\|U_{1} \Sigma_{1} y+b\right\|^{2} \leq c^{2}$. Since $U$ is unitary, multiplying by $U^{H}$ preserves norm, so the last inequality is equivalent to

$$
\left\|\left[\begin{array}{c}
U_{1}^{H} \\
U_{2}^{H}
\end{array}\right] U_{1} \Sigma_{1} y+\left[\begin{array}{c}
U_{1}^{H} \\
U_{2}^{H}
\end{array}\right] b\right\|^{2} \leq c^{2} .
$$

In its turn, this is equivalent to

$$
\left\|\begin{array}{c}
\Sigma_{1} y+U_{1}^{H} b \\
U_{2}^{H} b
\end{array}\right\|^{2} \leq c^{2} .
$$


This can be rewritten as

$$
\left\|\Sigma_{1} y+U_{1}^{H} b\right\|^{2} \leq c^{2}-\left\|U_{2}^{H} b\right\|^{2}
$$

So on the one hand, if inequality (7) has a solution, then that solution fits the parameterization given in Equations (9) - (11) and condition (8) is satisfied. On the other hand, if condition (8) is satisfied and first $w$ and $z$ and then $y$ and finally $x$ are picked according to the parameterization given in Equations $(9)-(11)$, then this $x$ provides a solution to inequality (7).

\subsection{Constant Matrix Test}

If we set $M:=\left[G_{21}, G_{22}\right]$, then the condition $e_{y}=0$ can be written as

$$
M\left\{\begin{array}{c}
\xi \\
\beta
\end{array}\right\}=e_{y}^{o} .
$$

This provides the first necessary condition for the existence of $\beta$ with $\|\beta\| \leq 1$ and $\xi$ for which $e_{y}=0$; namely:

$$
e_{y}^{o} \in \operatorname{Im}(M)
$$

Observe that if $V$ is invertible, then condition (16) is true, since $M$ has full row rank. Physically, this means that if the noise model at output is allowed to influence all output channels, then any output signal can be validated (without any help from $\Delta$ and/or $\epsilon$ ) if the noise is not constrained by a bound. Also, condition (16) is necessary and sufficient for the existence of $\beta \in \mathcal{C}^{n_{\epsilon}+n_{\nu}}$ and $\xi$ for which $e_{y}=0$. The remainder of this discussion is aimed at finding a condition to insure that $\|\beta\| \leq 1$.

If condition (16) is satisfied, then Equation (15) is solvable, and a complete parameterization of the solutions is given by

$$
\left\{\begin{array}{c}
\xi \\
\beta
\end{array}\right\}=M^{+} e_{y}^{o}+N_{M} \theta,
$$

where $N_{M}$ is a matrix whose columns form a basis for $\operatorname{Ker}(M)$, and the parameter $\theta$ is arbitrary. The notation $(\cdot)^{+}$denotes the Moore-Penrose pseudo-inverse of $(\cdot)$. 
Notation: Let $a$ be a vector defined by an equation of the form $a=A x+B y+\cdots$, and let $b$ be a subvector of $a$. Then by $(A)_{b},(B)_{b}$, etc., we are referring to those submatrices of $A, B$, etc., containing the complete rows of the respective matrices so that $b=(A)_{b} x+(B)_{b} y+\cdots$.

Equation (17) then implies that

$$
\beta=\left(M^{+}\right)_{\beta} e_{y}^{o}+\left(N_{M}\right)_{\beta} \theta
$$

and the problem is to characterize those $\theta$ for which $\|\beta\| \leq 1$.

This can be done by a direct application of Lemma 1. First perform an SVD of $\left(N_{M}\right)_{\beta}$ as in Lemma 1:

$$
\left(N_{M}\right)_{\beta}=\left[\begin{array}{ll}
T_{1} & T_{2}
\end{array}\right]\left[\begin{array}{cc}
\Sigma_{1} & 0 \\
0 & 0
\end{array}\right]\left[\begin{array}{c}
U_{1}^{H} \\
U_{2}^{H}
\end{array}\right]
$$

Then, by Lemma 1, Equation (18) can be solved for $\beta$ with $\|\beta\| \leq 1$ if and only if

$$
\left\|T_{2}^{H}\left(M^{+}\right)_{\beta} e_{y}^{o}\right\| \leq 1
$$

Then, the general solution to $\|\beta\| \leq 1$ subject to Equation (18) is given by

$$
\begin{aligned}
\theta & =U_{1} \gamma+U_{2} \psi \\
\gamma & =\Sigma_{1}^{-1}\left(\phi-T_{1}^{H}\left(M^{+}\right)_{\beta} e_{y}^{o}\right)
\end{aligned}
$$

where $\psi \in \mathcal{C}^{n_{\psi}}$ is arbitrary, and $\phi \in \mathcal{C}^{n_{\phi}}$ is any vector with

$$
\|\phi\| \leq b_{o}:=\sqrt{1-\left\|T_{2}^{H}\left(M^{+}\right)_{\beta} e_{y}^{o}\right\|^{2}} .
$$

In light of the definition in Equation (23), the necessary condition in (20) implies $b_{o} \geq 0$.

Lemma 2 (Constant Matrix Test):

With the context and notation established in the previous paragraphs, the following are equivalent:

1. There exists $(\xi, \beta),\|\beta\| \leq 1$ such that $y=G_{21} \xi+G_{22} \beta+G_{23} r$.

2. Conditions (16) and (20) hold. 
If these conditions are satisfied, then all such triples $(\xi, \epsilon, \nu)$ are given by the parameterization

$$
\left\{\begin{array}{c}
\xi \\
\beta
\end{array}\right\}=\left\{\begin{array}{c}
\xi_{o} \\
\beta_{o}
\end{array}\right\}+\Omega\left\{\begin{array}{c}
\phi \\
\psi
\end{array}\right\}
$$

where

$$
\begin{aligned}
\Omega & :=N_{M} U\left[\begin{array}{cc}
\Sigma_{1}^{-1} & 0 \\
0 & I_{\Omega}
\end{array}\right] \\
\left\{\begin{array}{l}
\xi_{o} \\
\beta_{o}
\end{array}\right\} & :=\left[M^{+}-N_{M}\left(\left(N_{M}\right)_{\beta}\right)^{+}\left(M^{+}\right)_{\beta}\right] e_{y}^{o}
\end{aligned}
$$

and where $\psi$ is arbitrary, and $\phi$ satisfies inequality (23).

Remark: The number of free parameters $(\phi, \psi)$ as defined by Equation (24) is given by $n_{\phi}+n_{\psi}=\operatorname{dim}(\operatorname{Ker}(M))$, and the dimension of the identity matrix, $I_{\Omega}$, is $n_{\phi}+n_{\psi}-$ $\operatorname{rank}\left(N_{M}\right)_{\beta}$.

\section{Proof of Lemma 2:}

The existence of a triple $(\xi, \epsilon, \nu)$ with the desired properties is equivalent to the solvability of Equation (15) with $\|\beta\| \leq 1$. We have already seen that Equation (15) can be solved with $\|\beta\| \leq 1$ if and only if conditions (16) and (20) hold, and all such solutions are parameterized by Equations $(21)-(23)$. When a value of $\theta$ as given by the parameterization in Equations $(21)-(23)$ is substituted in Equation (17) to eliminate $\theta$ and then $\gamma$, the expression for the triple $(\xi, \epsilon, \nu)$ given Equation (24) results.

Lemma 2 gives a test resulting in either a yes or no answer. It is only concerned with testing the richness of the a priori LFT uncertainty structure and chosen levels of measurement noise and disturbance allowance against a given set of measured input and output data. Whether such $\xi$ can be generated through the LFT uncertainty and how large it must be remains to be seen. If the test in Lemma 2 fails, then the model is invalidated either due to overly restricted levels of noise and/or disturbance and/or insufficiently rich uncertainty LFT structure. What course of action should be taken if the Lemma 2 test fails is not considered in this paper. Of course, at this point, increasing the magnitude of the LFT uncertainty bound will not help. 
Suppose the conditions of Lemma 2 are satisfied. Indeed, Lemma 2 gives a parameterization of the set of all $\xi$ that produces zero output error. That $\xi$ is given by this parameterization provides a necessary condition that $\xi$ be a signal in a model validated robust control design model. For sufficiency, $\xi$ must also satisfy the $G-\Delta$ feedback conditions

$$
\begin{aligned}
& \xi=\Delta \eta \\
& \eta=G_{11} \xi+G_{12} \beta+G_{13} r
\end{aligned}
$$

Since $\eta$ can be readily computed from Equation (26) for a given $\xi$ and $\beta$, we group Equations (24) and (26) as follows:

$$
\left\{\begin{array}{l}
\eta \\
\xi
\end{array}\right\}=\left\{\begin{array}{l}
\eta_{o} \\
\xi_{o}
\end{array}\right\}+\left[\begin{array}{cc}
G_{11} & G_{12} \\
I_{n_{\xi}} & 0_{n_{\xi} \times\left(n_{\epsilon}+n_{\nu}\right)}
\end{array}\right] \Omega\left\{\begin{array}{l}
\phi \\
\psi
\end{array}\right\}
$$

Here,

$$
\eta_{o}:=\left[\begin{array}{ll}
G_{11} & G_{12}
\end{array}\right]\left\{\begin{array}{l}
\xi_{o} \\
\beta_{o}
\end{array}\right\}+G_{13} r,
$$

and the norm of $\phi$ is subject to condition (23). Equation (27) characterizes the set of all $(\xi, \eta)$ vectors that produces zero output error. Of course, this set may be further constrained by the uncertainty structure given by Equation (25), which motivates the next lemma.

Consider a basic fact from linear algebra as noted earlier in [8]:

Lemma 3:

If $u \in \mathcal{C}^{m}, v \in \mathcal{C}^{n}, v \neq 0$, then there exists $A \in \mathcal{C}^{m \times n}$ such that $A v=u$, and $\bar{\sigma}(A)=\frac{\|u\|}{\|v\|}$.

Remark: If $A v=u$, then $\|A\|=\bar{\sigma}(A) \geq \frac{\|u\|}{\|v\|}$, so this lemma is asserting that an $A$ of the minimal possible norm does exist which maps $v$ onto $u$. This will find application in this paper in demonstrating the existence of model validating blocks $\Delta_{i}$ of minimal possible norm.

\section{$3.2 \quad$ Full Complex Blocks Only}

We now work toward determining when there exist model validating $\Delta \in \mathcal{D}$ with the implicit assumption of structured, full complex blocks only for $\Delta$ in this section. To this end, we 
start with uncertainty signals $\xi$ and $\eta$ which satisfy Equation (27) and look for $\Delta$ for which Equation (25) is also satisfied. Partition $\xi$ and $\eta$ into components corresponding to the block structure of $\Delta$ so that Equation (25) could be written as

$$
\xi_{i}=\Delta_{i} \eta_{i}, \quad i=1, \ldots, \tau
$$

Since, for each $i=1, \ldots, \tau, \xi_{i}=\Delta_{i} \eta_{i}$, one can never have both $\xi_{i} \neq 0$ and $\eta_{i}=0$ (cf. Lemma 3). However, the parameterization in Equation (27) does not guarantee that these conditions never occur. To have some terminology to use to indicate that we are excluding this possibility, we make the following definition:

\section{Definition (D-realizable):}

A signal pair $(\xi, \eta)$ will be called $\mathcal{D}$-realizable if, for each $i=1, \ldots, \tau$, either $\xi_{i}=0$ or $\eta_{i} \neq 0$.

Satisfaction of the test in Lemma 2 allows a cancellation of the nominal output error by a combination of the fictitious signals from the uncertainty block and a norm bounded exogenous disturbance while it will be shown that Lemma 3 guarantees that a structured, full complex uncertainty always exists for any $\mathcal{D}$-realizable pair $(\xi, \eta)$ which satisfy Equation $(27)$. We state an existence condition and a parameterization of all model validating uncertainty sets as follows:

Theorem 1 (structured, full complex blocks):

Suppose the conditions given in Lemma 2 are satisfied. Then, all model validating sets of plants are given by

$$
\mathcal{P}_{W \phi \psi}:=\left\{F_{u}(P, \Delta), \Delta \in \mathcal{D}_{W}\right\}
$$

where $\psi \in \mathcal{C}^{n_{\psi}}, \phi \in \mathcal{C}^{n_{\phi}},\|\phi\| \leq b_{o}, W:=\operatorname{diag}\left(w_{1} I_{n_{1}}, \ldots, w_{\tau} I_{n_{\tau}}\right)$ is any diagonal complex matrix whose diagonal elements satisfy

$$
\left|w_{i}\right| \geq \frac{\left\|\xi_{i}\right\|}{\left\|\eta_{i}\right\|}, \quad i=1, \ldots, \tau
$$


and the $(\xi, \eta)$ pair parameterized by $\phi$ and $\psi$ as given in Equation $(27)$ is $\mathcal{D}$-realizable.

\section{Proof of Theorem 1:}

It is first demonstrated that each $\mathcal{P}_{W \phi \psi}$ described in the statement of Theorem 1 is a model validating set. Since the conditions of Lemma 2 are satisfied, the $\xi$ given by Equation (27) combines with an exogenous disturbance $\beta$ for which $\|\beta\| \leq 1$ to produce $e_{y}=0$. Therefore, $\mathcal{P}_{W \phi \psi}$ will be model validating if there exists $\Delta \in \mathcal{D}_{W}$ for which Equation (28) is satisfied. We construct this $\Delta$ block by block. If $\xi_{i}=0$, then $\Delta_{i}$ and $\Delta_{B i}$ may be taken to be 0 and $\bar{\sigma}\left(\Delta_{B i}\right)=0$. If $\xi_{i} \neq 0$, then since $(\xi, \eta)$ is a $\mathcal{D}$-realizable pair, $\eta_{i} \neq 0$ and by Lemma 3 , there exists a $\Delta_{i}$ with $\Delta_{i} \eta_{i}=\xi_{i}$ and $\bar{\sigma}\left(\Delta_{i}\right)=\left\|\xi_{i}\right\| /\left\|\eta_{i}\right\|$. This means that if $w_{i} \neq 0$ and $\Delta_{B i}:=\left(1 / w_{i}\right) \Delta_{i}$, then $\bar{\sigma}\left(\Delta_{B i}\right) \leq 1$ (if $w_{i}=0, \Delta_{B i}$ may be chosen arbitrarily with $\left.\bar{\sigma}\left(\Delta_{B i}\right) \leq 1\right)$. It follows that if $\Delta_{B}:=\operatorname{diag}\left(\Delta_{B 1}, \Delta_{B 2}, \cdots, \Delta_{B \tau}\right)$, then $\bar{\sigma}\left(\Delta_{B}\right) \leq 1$. Then $\Delta=\Delta_{B} W \in \mathcal{D}_{W}$, so $\mathcal{P}_{W \phi \psi}$ is model validating.

Now let $\mathcal{P}_{W}$ be an arbitrary model validating set. Let $\Delta \in \mathcal{D}_{W}$ zero the output error for some $\beta$ where $\|\beta\| \leq 1$. Then there exist signals $\xi$ and $\eta$ which satisfy Equations (25) and (26) such that $\xi$ zeros out the error with some $\beta$ where $\|\beta\| \leq 1$. Then by Lemma 2 , there exist $\phi$ satisfying condition (23) and $\psi$ such that $\xi$ and $\eta$ are expressed in terms of $\phi$ and $\psi$ by Equation $(27)$. Since $\xi_{i}=\Delta_{i} \eta_{i}$ for all $i=1, \ldots, \tau$, the pair $(\xi, \eta)$ must be $\mathcal{D}$-realizable, and $\left\|\xi_{i}\right\|=\left\|\Delta_{i} \eta_{i}\right\| \leq \bar{\sigma}\left(\Delta_{i}\right)\left\|\eta_{i}\right\| \leq\left|w_{i}\right|\left\|\eta_{i}\right\|$, the last inequality holding because $\Delta \in \mathcal{D}_{W}$. This establishes that $\mathcal{P}_{W}=\mathcal{P}_{W \phi \psi}$.

For practical applications where the $\mathcal{D}$-realizable assumption is typically satisfied, Lemma 2 can be viewed as a necessary and sufficient condition for the existence of a model validating set for an LFT with only structured full complex blocks. The issue that remains is how large must the uncertainty size be for model validation, which is addressed in Theorem 1. For fixed parameters $\phi$ and $\psi$, the smallest model validating uncertainty set is given by $\left|w_{i}\right|=\frac{\left\|\xi_{i}\right\|}{\left\|\eta_{i}\right\|}, i=1, \ldots, \tau$. This is the minimum norm model validating result reported in [8] and any choice of uncertainty set bounds $\left\{w_{i}, i=1, \ldots, \tau\right\}$ for which $\left|w_{i}\right|<\inf \underset{\|\phi\| \leq b_{o}}{\psi} \frac{\left\|\xi_{i}\right\|}{\left\|\eta_{i}\right\|}$ for some $i$, will not be a model validating set. 


\subsection{Repeated Scalar Blocks}

For a more general uncertainty structure which includes repeated and/or real scalar blocks, we assume for convenience, that all repeated scalar blocks are grouped into the first $r$ blocks in $\Delta$ as given in Equation (1). Since $\Delta$ is further restricted, the conditions in Lemma 2 or Theorem 1 becomes only a necessary condition for model validation. So, with repeated scalar blocks, we ask whether, among all $\mathcal{D}$-realizable pairs $(\xi, \eta)$ satisfying Equation $(27)$ with $\phi$ subject to the norm condition (23), a pair exists for which a $\Delta$ of the form given in Equation (1) also exists so that Equation (25) is satisfied?

First, note that given any such $\mathcal{D}$-realizable pair $(\xi, \eta), \Delta_{r+1}, \ldots, \Delta_{\tau}$ always exist by Lemma 3, so we need to consider only the existence of the first $r$ blocks, $\delta_{1} I_{n_{1}}, \ldots, \delta_{r} I_{n_{r}}$ where $\delta_{i} \in \mathcal{F}_{i}$. Let us denote $\bar{n}:=\sum_{i=1}^{r} n_{i}=\bar{m}:=\sum_{i=1}^{r} m_{i}$. A model validating uncertainty set exists for a system with repeated scalar block if and only if there exists $\delta_{i} \in \mathcal{F}_{i}, i=1, \ldots, r$, $\psi, \phi,\|\phi\| \leq b_{o}$ such that

$$
\xi_{i}=\delta_{i} \eta_{i}, \quad i=1, \ldots, r
$$

where from Equation (27):

$$
\begin{aligned}
\xi_{i} & =\xi_{o, i}+\Omega_{i}\left\{\begin{array}{c}
\phi \\
\psi
\end{array}\right\} \\
\eta_{i} & =\eta_{o, i}+\left[\begin{array}{ll}
G_{11} & \left.G_{12}\right]_{i} \Omega
\end{array}\left\{\begin{array}{c}
\phi \\
\psi
\end{array}\right\}\right.
\end{aligned}
$$

The subscript $i$ indicates that the correct blocks of rows have been selected for Equations (32) and (33) to make sense in the context of Equation (27) and the decompositions of $\xi$ and $\eta$.

The condition in Equation (31) can be seen as a collinearity condition in the vector space $\mathcal{C}^{n_{i}}$ with coefficients from the field $\mathcal{F}_{i}$. Consequently, a measure of distance between two subspaces can be used (see for example [12])

$$
\operatorname{dist}^{\left(\mathcal{F}_{i}\right)}\left(\xi_{i}, \eta_{i}\right):=\left\|P_{\xi_{i}}^{\left(\mathcal{F}_{i}\right)}-P_{\eta_{i}}^{\left(\mathcal{F}_{i}\right)}\right\|
$$

where $P_{\xi_{i}}^{\left(\mathcal{F}_{i}\right)}$ and $P_{\eta_{i}}^{\left(\mathcal{F}_{i}\right)}$ denote orthogonal projections onto the subspaces spanned over the 
field $\mathcal{F}_{i}$ by the single vectors $\xi_{i}$ and $\eta_{i}$, respectively. As an illustration, in the case of one dimensional subspace in $\mathcal{R}^{3}$, the distance measure as given by Equation (34) reduces to the sine of the angle between the two lines. We summarize our results as follows:

Theorem 2 (with repeated scalar block):

(a) Suppose the conditions in Lemma 2 are satisfied. Then a model validating set exists with $\Delta_{i}=\delta_{i} I_{n_{i}}, i=1, \ldots, r, \delta_{i} \in \mathcal{F}_{i}$ if and only if there exists $\psi$, and $\phi$ with $\|\phi\| \leq b_{o}$ such that the $(\xi, \eta)$ pair as given in Equation $(27)$ is $\mathcal{D}$-realizable and for each $i=1, \ldots, r$,

$$
\text { either } \xi_{i}=0 \quad \text { or } \quad \operatorname{dist}^{\left(\mathcal{F}_{i}\right)}\left(\xi_{i}, \eta_{i}\right)=0
$$

where $\xi_{i}$ and $\eta_{i}$ are given by Equations (32) and (33).

(b) Furthermore, if a model validating set exists, then all such sets are given by Equation (29) where $W$ is any diagonal complex matrix whose diagonal elements satisfy condition (30) and the $(\xi, \eta)$ pair parameterized by $\phi$ and $\psi$ as given in Equation $(27)$ is $\mathcal{D}$-realizable and satisfies condition (35).

In order to prove Theorem 2, we first introduce a lemma.

\section{Lemma 4:}

For each fixed $i$, condition (35) holds if and only if there exists a $\delta_{i} \in \mathcal{F}_{i}$ such that $\xi_{i}=\delta_{i} \eta_{i}$

\section{Proof of Theorem 2:}

First, suppose that there exist $\psi$ and $\phi$ with $\|\phi\| \leq b_{o}$ such that the $(\xi, \eta)$ pair as given in Equation (27) is $\mathcal{D}$-realizable and condition (35) holds. Then by Theorem 1, any set $\mathcal{P}_{W \phi \psi}$ as given in Equation (29) with $W$ satisfying condition (30) is model validating. Let $\Delta \in \mathcal{D}_{W}$ be a model validating uncertainty model. The key properties of $\Delta$ are that, for each $i=1, \ldots, \tau, \| \Delta_{i}|| \leq\left|w_{i}\right|$ and $\xi_{i}=\Delta_{i} \eta_{i}$. Because of condition (35), Lemma 4 tells us that we can replace each $\Delta_{i}$ for $i=1, \ldots, r$ by a matrix of the form $\delta_{i} I_{n_{i}}$ with $\delta_{i} \in \mathcal{F}_{i}$ and still have $\xi_{i}=\Delta_{i} \eta_{i}$. Also, since $\xi_{i}=\delta_{i} \eta_{i}$ and $W$ satisfies condition (30), we still have $\left\|\Delta_{i}\right\| \leq\left|w_{i}\right|$ with this new $\Delta_{i}$. This establishes the "if" part of Theorem 2(a), and shows 
that every model validating set with the specified repeated scalar blocks has the form shown in Theorem 2(b).

Now suppose that a model validating set $\mathcal{D}$ exists which contains a model validating uncertainty model $\Delta$ with $\Delta_{i}=\delta_{i} I_{n_{i}}, i=1, \ldots, r, \delta_{i} \in \mathcal{F}_{i}$. Theorem 1 tells us that $\mathcal{D}$ must satisfy all of the conclusions of Theorem 2(b) except possibly the condition (35). However, since for each $i=1, \ldots, r, \Delta_{i}=\delta_{i} I_{n_{i}}$, it follows from Lemma 4 that condition (35) is satisfied. This completes the proof that every model validating set falls under the description given in Theorem 2(b), and completes the "only if" part of the proof of Theorem 2(a).

\section{Uncertainty Bound Tradeoff}

With the parameterization of all model validating uncertainty sets given by Theorems 1 and 2, a controls engineer still faces the issue of how to utilize the remaining freedom. Specifically, one may ask: can we find a smallest set from the given parameterization of all model validating uncertainty sets? This begs the issue of which "smallest set" for this underdetermined problem. For a single uncertainty block problem, a smallest-norm model validating uncertainty appears to be a physically reasonable uncertainty bound based on Ockham's razor argument in modeling physical systems. However, for problems with a general LFT uncertainty structure which can be viewed as a multi-objective problem, an uncertainty bound having a smallest norm may not have any concrete physical justification. This is because for problems with multiple uncertainty blocks, their relative numerical values do not necessarily indicate their relative physical significance. For example, in robust stability [13], the determination of whether a controller guaranteeing robust stability exists or not may depend more strongly on the distribution of the uncertainty bounds over a given set of uncertainty components than on the size of largest uncertainty component.

In this section, we outline two algorithms based on constrained nonlinear optimization to determine a smallest uncertainty set. One starting point for an optimization would be for the designer to select an augmented nominal plant model, $P$, a noise allowance filter, $V$, 
and a matrix, $W$, of desired levels of uncertainty in the blocks. The important feature of $W$ is that the relative sizes of the $w_{i}$ reflect the designer's wishes as to the relative size of the uncertainty levels in the different blocks. A smallest uncertainty set search algorithm is outlined as follows:

1. select $(P, \mathcal{D}, V)$

2. test feasibility of $(P, \mathcal{D}, V)$ against $(r, y)$.

3. select $W$

4. find smallest $x$ such that $\mathcal{P}_{(x W)}$ is model validating

If the feasibility test in Step 2 fails, redo Step 1 until it passes. For uncertainty structure with only full complex blocks, Step 2 is simply a constant matrix test given in Section 3.1, otherwise, Step 2 can be included as a constraint in Step 4 (see Remark in Section 4.3). Using $\mathcal{P}_{(x W)}$ from Step 4 and a chosen performance weight, a robust controller can be obtained by $\mu$ synthesis. If the closed loop system is determined to be unsatisfactory or suspected to be unrealistic, a new model validating set can be obtained by reselecting the weights $W$ in Step 3 and repeating Step 4. In Step 4, if the chosen $W$ is a desired set of weights and the computed smallest $x$ is less than 1 , then the postulated robust control design model, $\mathcal{P}_{W}$ can be considered to be model validating.

As a prelude to trying to optimize uncertainty levels during a tradeoff, the necessary conditions (16) and (20) for the existence of model validating sets, should be checked. If all of the output channels are being modeled as having noise in them, so that the $V$ matrix is non-singular, then the matrix $M$ is of full rank, and condition (16) always holds. However, if the diagonal matrix $V$ does have zeros on the diagonal (or, for the general case $V \geq 0$ for fully populated $V$ ), then a check on condition (16) can be made by first performing an SVD on $M: M=U_{M} \Sigma_{M} V_{M}^{H}$ where $U_{M}$ and $V_{M}$ are unitary and $\Sigma_{M}$ is a non-negative real diagonal matrix of the same shape as $M$ whose diagonal elements are in decreasing order. Condition (16) also holds if $M$ is full rank which is equivalent to $\Sigma_{M}$ having no zero rows. 
If $M$ is rank deficient, then partition $U_{M}=\left[U_{M 1}, U_{M 2}\right]$ where the block $U_{M 1}$ corresponds to the non-zero rows of $\Sigma_{M}$ and the block $U_{M 2}$ corresponds to the zero rows of $\Sigma_{M}$. Then a necessary and sufficient condition in the case of rank deficient $M$ for condition (16) to hold is that $U_{M 2} e_{y}^{o}=0$. To verify condition (20), the SVD of $M$ is computed in the form:

$$
M=\left[\begin{array}{ll}
U_{M 1} & U_{M 2}
\end{array}\right]\left[\begin{array}{cc}
\Sigma_{M 1} & 0 \\
0 & 0
\end{array}\right]\left[\begin{array}{c}
V_{M 1}^{H} \\
V_{M 2}^{H}
\end{array}\right]
$$

In this decomposition, $U=\left[\begin{array}{ll}U_{M 1} & U_{M 2}\end{array}\right]$ and $V=\left[\begin{array}{ll}V_{M 1} & V_{M 2}\end{array}\right]$ are unitary, $\Sigma_{M 1}$ is diagonal and nonsingular, and the block matrix partitionings are conformal. Then, in Equation (17), $M^{+}=V_{M 1} \Sigma_{M 1}^{-1} U_{M 1}^{H}$ and $N_{M}$ can be taken to be $V_{M 2}$. Then, $\left(N_{M}\right)_{\beta}$ is decomposed as in Equation (19), and all of the components are at hand to execute the test in condition (20).

\subsection{Full Complex Blocks}

The idea is to normalize all uncertainties using desired levels of uncertainty in the uncertainty blocks, and then seek the smallest model validating scaled set. Specifically, we propose using nonlinear constrained optimization with $\psi$ and $\phi$ as the design parameters to find a minimal positive $x$ such that $\mathcal{P}_{(x W)}$ is a model validating set.

By Theorem $1, \mathcal{P}_{(x W)}$ is a model validating set if there exist $\psi$ and $\phi$ with $\|\phi\| \leq b_{o}$ such that

$$
x\left|w_{i}\right| \geq \frac{\left\|\xi_{i}\right\|}{\left\|\eta_{i}\right\|}, \quad i=1, \ldots, \tau
$$

where the vectors $\xi$ and $\eta$ calculated from $\psi$ and $\phi$ in Equation (27) form a $\mathcal{D}$-realizable pair. This implies that $\mathcal{P}_{(x W)}=\mathcal{P}_{(x W) \psi \phi}$. By squaring and clearing fractions, the previous inequality can be combined with the $\mathcal{D}$-realizability condition in the single inequality in Equation (39). This also has the advantage of being a polynomial in $x$ and the components of $\xi$ and $\eta$. The optimization problem can now be stated:

A Smallest Set (Full Complex Blocks):

$$
\min _{\psi, \phi, x} x
$$


subject to

$$
\begin{aligned}
\left\|\xi_{i}\right\|^{2}-x^{2}\left|w_{i}\right|^{2}\left\|\eta_{i}\right\|^{2} & \leq 0, \quad i=1, \ldots, \tau \\
x & \geq 0 \\
\|\phi\| & \leq b_{o}
\end{aligned}
$$

where $\xi_{i}$ and $\eta_{i}$ are given by Equations (32) and (33).

For the special case where the noise is known or given, the parameter $\phi$ is unbounded and Equation (41) is no longer needed. This is the case derived earlier as a minimum norm model validating solution [8]. For the class of problems where the uncertainty structure is such that all $\eta_{i}$ 's are completely determined by input and output data (for example additive, multiplicative, additive feedback, etc), a feasible set in the above optimization algorithm is convex for any given $x$. Hence for this special class of uncertainty structure, the model validation test (with $x=1$ ) can be seen as a convex feasibility problem as noted earlier [4].

\subsection{Repeated Scalar Blocks}

For the case with repeated and/or real scalar blocks, an optimization algorithm similar to full complex block case but with the additional collinearity condition is proposed. Similarly, by Theorem $2, \mathcal{P}_{(x W)}$ is a model validating set if there exist $\psi$ and $\phi$ with $\|\phi\| \leq b_{o}$ such that condition (35) is satisfied where $\xi_{i}$ and $\eta_{i}$ is a $\mathcal{D}$-realizable pair parameterized by Equations (32) and (33). Instead of using the distance condition in Equation (35) to guarantee existence, Equation (31) is used. The tradeoff is that Equation (35) leads to a quartic in the design variables while Equation (31) leads to only a quadratic at the expense of additional variables, $\delta_{1}, \ldots, \delta_{r}$. Note that the collinearity condition for the set of $r$ repeated scalar blocks and the $\mathcal{D}$-realizability condition leads to a simplification of the first $r$ set of inequality constraints in Equation (39). The optimization problem can now be stated:

A Smallest Set (With Repeated and/or Real Scalar Blocks): 


$$
\min _{\psi, \phi, \delta_{1}, \ldots, \delta_{r}, x} x
$$

subject to

$$
\begin{aligned}
\delta_{i} & \in \mathcal{F}_{i} \quad i=1, \ldots, r \\
\xi_{i} & =\delta_{i} \eta_{i}, \quad i=1, \ldots, r \\
\left|\delta_{i}\right|^{2}-x^{2}\left|w_{i}\right|^{2} & \leq 0, \quad i=1, \ldots, r \\
\left\|\xi_{i}\right\|^{2}-x^{2}\left|w_{i}\right|^{2}\left\|\eta_{i}\right\|^{2} & \leq 0, \quad i=r+1, \ldots, \tau \\
x & \geq 0 \\
\|\phi\| & \leq b_{o}
\end{aligned}
$$

where $\xi_{i}$ and $\eta_{i}$ are given by Equations (32) and (33).

Remark: In Section 4 constant matrix tests were given which were necessary, but not sufficient for the existence of a model validating set for the case that some uncertainty blocks have repeated scalar blocks. Such a sufficient condition is found by the existence of a feasible point in the preceding optimization problem. In particular, in order to have a model validating set in this case, it is necessary to satisfy constraints (43) and (44). The existence of a feasible point is also sufficient for satisfying $\mathcal{D}$-realizability. Note also that the above constraints involve polynominals in $\phi, \psi, \delta_{1}, \ldots, \delta_{r}, x$ which are at most quartic.

To summarize, the above optimization algorithm has the following physical significances. The cost in Equation (42) represents a positive scaling factor of the normalized (by user provided desirable weights) uncertainty norm bounds for each component. Inequalities (45) and (46) represent the scaled bounds on $r$ repeated scalar uncertainties and the $\tau-r$ nonrepeated full complex uncertainty bounds, respectively. A violation of these inequalities implies that $x\left|w_{i}\right|$ is not an upper bound on the ratio of signal norms, i.e., it fails as an uncertainty bound. Clearly, inequalities (45) and (46) will more likely be satisfied with larger weights $x\left|w_{i}\right|$, which makes intuitive sense. The collinearity condition in Equation (44) represents the necessary structural constraints due to the repeated scalar uncertainties. 
Inequality (47) is the non-negative condition on the uncertainty scale factor. Note that $x \geq 1$ indicates that the current scaling makes the uncertainty bounds larger or equal to the a priori target while $x<1$ indicates that there exists a smaller (for every component) model validating set than the a priori target. Finally, inequality (48) represents a noise allowance.

\subsubsection{Real Parametric Uncertainties}

In the uncertainty optimization algorithm such as Equations (42)-(48), optimistic (to an unknown degree) levels of non-parametric uncertainty bounds are expected since the uncertain parameters are free to be a function of frequency although subjected to fixed lower and upper bounds. In $\mu$ analysis and synthesis, the uncertainty is only restricted to be norm bounded and stable and therefore will lead to conservative results. The net result is that an optimistic prediction of non-parametric uncertainty bounds will be compensated to some unknown degree by the conservative prediction from $\mu$ analysis and synthesis.

Suppose the repeated and/or real scalar blocks are independent of frequencies, i.e., the parameter is unknown but constant and we wish to accomodate this by specifying lower and upper bounds to design for anticipated changes. Of course parameter estimation can precede the specification of the above bounds. Instead of jointly optimizing a set of uncertain parameters with non-parametric uncertainties as shown in Equations (45) and (46), we propose solving for the smallest non-parametric uncertainties subject to a priori allowance in exogenous disturbance/noise and parametric uncertainties. This can be implemented by eliminating the scale factor $x$ in Equation (45). A practical justification for this approach is that (physical) parameters are generally better modeled then non-parametric uncertainties since the latter type typically originates from complex dynamics with many details which are difficult and subsequently not explicitly modeled, viz "unmodeled dynamics". Therefore, to determine a smallest model validating uncertainty set for problems with parametric uncertainties, we propose (a) introducing sufficient non-parametric uncertainties to satisfy model validation conditions (constant matrix tests) and then (b) introducing varying levels of parametric uncertainty allowances to tradeoff with smallest non-parametric uncertainty 
levels.

\section{$5 \quad$ Illustrative Example}

\subsection{Simulated System and Nominal Model}

Consider a simulated (true) system composed of two lumped masses-spring-dashpot with 2 inputs and 2 outputs which are fixed at two ends as given in [7]. For the purpose of this study, suppose the nominal plant model is chosen to consist of only the first mode to reflect a reduced order model. The neglected second structural mode reflects unmodeled dynamics. The nominal plant model consisting of the single mode is assumed to have parametric uncertainties in the frequency and damping. The two structural resonances corresponds to approximately 2.9 and 5.0 Hertz with damping ratios of 6 and 10 percents respectively.

Figure 2 shows the Bode plot of the true system, nominal plant model, and their difference. Notice that the largest differences occur at the structural resonances. The first peak in the difference is due to the parametric uncertainty in the first mode whereas the second peak is due to the unmodeled dynamics corresponding to the truncated second mode.

With the chosen nominal plant and the uncertainty structure, the system interconnection is constructed. The frequency and damping parametric uncertainties are modeled in discrete time state-space while the non-parametric additive uncertainty is between the control inputs and plant outputs. The frequency and damping parametric uncertainty in the first mode as represented in the discrete time domain leads to a repeated parametric uncertainty of order 2. A set of 1024 time points are assumed sampled at $50 \mathrm{~Hz}$. The simulated measurement noise and input test signals are generated by filtering independent uniform random signals through two different wideband filters. The simulated output signal to noise ratio ranged from 1 to $10^{3}$ over bandwidth of interest. 


\subsection{Parametric Study}

Consider the problem of finding the smallest unmodeled dynamics uncertainty for model validation subject to bounded noise and parametric uncertainty. A smallest set algorithm defined by Equations (42) - (48) is applied with a modification to Equation (45) as discussed in Section 4.2.1. Analytical sensitivity formulae were used with a constrained optimization routine in [14] based on a quasi-Newton algorithm. Since unlimited additive uncertainty is included, existence conditions given in Lemma 2 are satisfied. We consider nine cases (see Table 1) corresponding to a combination of 3 levels of parametric uncertainty allowance and 3 levels of output noise allowance. In the determination of a smallest model validating set, parametric uncertainties are allowed to be functions of frequency as long as they satisfy a constant norm bound over frequency. As discussed earlier, this will lead to an optimistic level of non-parametric model validating uncertainty. However, in the actual design of controllers, the a priori parametric norm bound is used for the robust control design model, instead of the smaller converged parametric uncertainty value.

\subsubsection{Output Noise Allowance}

As shown in Table 1, three levels of the output noise allowance are considered. The peak 2-norm of the true noise spectrum over the bandwidth of interest, 1 to $10 \mathrm{~Hz}$, is denoted by $\hat{V}_{\text {peak }}:=0.078 I_{2 \times 2}$. In Cases $1 \mathrm{a}, 2 \mathrm{a}$, and $3 \mathrm{a}$, a high noise allowance corresponding to $\hat{V}_{\text {peak }}$ is assumed, while a moderate noise level of $\hat{V}_{r m s}=\operatorname{diag}(.0321, .0332)$ is assumed in Cases $1 \mathrm{~b}$, $2 \mathrm{~b}$, and $3 \mathrm{~b}$. This root-mean-square over the above bandwidth is computed independently

for each output channel. Finally, a low noise allowance of $1 \%$ of $\hat{V}_{\text {peak }}$ is assumed in Cases 1c, $2 \mathrm{c}$, and 3c. In the actual model validation computations, since the allowed independent white noise, $\nu$, is bounded by unity, the noise filter is scaled by a factor of $\sqrt{2}$.

\subsubsection{Eigenvalue Uncertainty Allowance}

In Cases 1(a-c), an optimistically small level of parametric uncertainty allowance is assumed, Cases 2(a-c) represents a realistic or ball park estimate, and finally Cases 3(a-c) represents 
overly pessimistic levels of the actual parametric uncertainty levels. Given parametric uncertainty bounds

$$
\lambda\left(\delta_{1}, \delta_{2}\right)=.913 \pm .348 i+\delta_{1}+\delta_{2} i, \quad\left|\delta_{1}\right| \leq \bar{\delta}_{1} \quad\left|\delta_{2}\right| \leq \bar{\delta}_{2}
$$

where

$$
\lambda_{\text {true }}=(.918 \pm .358 i, .756 \pm .550 i)
$$

find smallest model validating additive uncertainty. In other words, given specific output noise allowance, to what extent does specified parametric uncertainty levels contribute in reducing the additive errors?

\subsection{Smallest Model Validating Non-parametric Uncertainty}

Figure 3 shows the smallest levels of unmodeled additive dynamics needed for model validation for corresponding levels of parametric uncertainty and noise allowances with the following observations:

- For Cases 1 (a-c) where a small level of parametric uncertainty allowance is given, the minimum model validating additive uncertainty levels show two peaks at the resonances. A large level of additive uncertainty is required to cover the parametric error in the first mode.

- In Cases 2 (a-c), a ball park level of parametric uncertainty allowance helps to eliminate the additive uncertainty peak in the neighborhood of first mode.

- An increase in the parametric uncertainty allowance as in Cases 3 (a-c) further reduces the additive uncertainty in the neighborhood of first mode. However, the minimum levels of additive uncertainty for model validation around the second mode frequencies did not drop significantly in spite of a large parametric uncertainty allowance. This is expected from a structural dynamics viewpoint whereby allowing changes in a modal parameter will not significantly affect other well separated structural resonances. 
- Increasing noise allowance (up to the true peak noise value) has a negligible effect on the level of minimum model validating additive uncertainty although a slight drop in the uncertainty levels are noted. A noise allowance level of at least $100 \times$ the peak noise was necessary to cover the entire output nominal error due to parametric uncertainty and unmodeled dynamics.

\subsection{Robust Performance Comparison}

Stable, low-order (up to 4 th order) rational functions are used to over bound the model validating additive uncertainties shown in Figure 3 for the three cases using a routine from [2]. Based on the three sets of fitted additive and corresponding parametric uncertainties, D-K Iterations with constant D-scales were used to design suboptimal $\mu$ and skewed- $\mu$ controllers, $K_{\mu}^{1}, K_{\mu}^{2}$, and $K_{\mu}^{3}$, and $K_{\mu_{s}}^{1}, K_{\mu_{s}}^{2}$, and $K_{\mu_{s}}^{3}$, corresponding to Cases 1 to 3 . The skewed- $\mu$ controllers were obtained by computing a scaled sequence of $\mu$ synthesis problems (see for example, [1], Chapter 8). The performance weight chosen is, $W_{p e r f}=2 I_{2 \times 2}$. All calculated lower and upper bounds were close which means that the bounds are a good estimate of the actual values. The skewed- $\mu$ controller represents the optimal worst case performance controller over each model validating set. Although the uncertainty parameters are assumed and computed as real numbers, for simplicity they were assumed to be complex perturbations in the controller synthesis so that it is biased (larger $\mu$ ) towards Case 2 and more against Case 3. The performance indices for the controllers are summarized in Table 2. Case 3 appears best (peak $\mu$, peak skewed- $\mu$ ), despite the assumption of $\delta_{i} \in C$. All three $\mu_{\text {peak }}$ occured around the first structural mode.

As a final comparison, the true closed loop response is computed for all six controllers. The maximum singular value frequency responses of the simulated (true) closed loop transfer function matrices are shown in Figure 4. All three $\mu$ controllers show equal performance on true model whereas Skewed- $\mu$ controllers performed differently $\left(K_{\mu_{s}}^{2}, K_{\mu_{s}}^{3}\right.$ show equal performance while $K_{\mu_{s}}^{1}$ gave poorer performance) Interestingly, this better performance (in 
terms of $\mu$, Skewed- $\mu$, True response) occurs in spite of the fact that the uncertainty set used in $K_{\mu_{s}}^{2}$ and $K_{\mu_{s}}^{3}$ were more conservative since parametric uncertainties were treated as complex in the controller synthesis and $K_{\mu_{s}}^{2}$ and $K_{\mu_{s}}^{3}$ assumes larger parametric uncertainties.

In summary, non-physical model validating sets (Cases 1,3) can produce as good (Case 3) or worse result (Case 1) than a physically correct set (Case 2). In this particular example, there appears to be a preference of parametric uncertainty over additive uncertainty for better robust performance. This may be related to the fact that the open loop response

shows a significantly larger first mode response than the second mode (see Figure 4) and the fact that the first mode is limited by parametric uncertainty which Case 1 practically ignores.

\section{Conclusions}

For models of physical systems where the uncertainty is described by a linear fractional transformation with unknown but bounded exogenous disturbances, conditions for the existence of a model validating set are derived. For the case with only structured full complex blocks, this condition is necessary and sufficient and can be readily tested. This test is actually a test on the richness of the a priori uncertainty structure enhanced by a frequency weighted unknown but bounded exogenous disturbances and measurement noise allowance. It is significant that this test applies for an arbitrary number of full complex blocks. For the more general case when repeated and/or real scalar uncertainties are also present, we have shown that an additional condition involving a collinearity test is required. Model validating sets of uncertainties are in general highly nonunique so that a useful design tool should provide an efficient way of trading off parametric, non-parametric and exogenous noise uncertainty levels. Based on a parametrization of all model validating sets, an optimization algorithm is proposed which seeks a smallest model validating set of non-parametric uncertainties while subject to fixed levels of parametric uncertainties and noise allowances.

\section{Acknowledgement}


The authors appreciate comments from Professor Gary Balas of University of Minnesota, which helped improve the paper.

\section{References}

[1] Skogestad, S., and Postlethwaite, I., Multivariable Feedback Control: Analysis and Design, John Wiley \& Sons Inc, New York, NY, 1996.

[2] Balas, G.J., Doyle, J.C., Glover, K., Packard, A., and Smith, R., $\mu$-Analysis and Synthesis Toolbox, MUSYN Inc., Minneapolis, MN, 1994.

[3] Smith, R.S., Doyle, J.C., "Model Validation: A connection between robust control and identification," IEEE Transactions on Automatic Control, vol 37, No 7, July 1992, pp.942-952.

[4] Smith, R.S., Dullerud, G., Rangan, S., and Poolla, K., " Model validation for dynamically uncertain systems," Mathematical Modeling of Systems, 1997, Vol. 3, No. 1, pp. $43-58$.

[5] Special Issue on System Identification and Control, IEEE Transactions on Automatic Control, v.37, n.7, pp. 899-1008, July 1992.

[6] Smith, R.S., "Model validation for robust control: an experimental process control application," Automatica, v.31,, pp. 1637-47, Nov. 1995.

[7] Kumar, A., and Balas, G.J., "A scaling approach to model validation in the $\mu$ framework," Proceedings of the American Control Conference, 1995, pp.693-697.

[8] Lim, K.B., Balas, G.J., and Anthony, T.C., "Minimum-norm model validating identification for robust control," AIAA Paper No. 96-3717.

[9] Lim, K.B., Cox, D.E., Balas, G.J., and Juang, J-N., "Validation of an experimentally derived uncertainty model," AIAA Journal of Guidance, Control, and Dynamics, Vol. 21, No. 3, pp. 485-492, 1998. 
[10] Davis, R.A., and Glover, K., "An application of recent model validation techniques to flight test data," Proceedings of the European Control Conference, 1995, Rome, Italy.

[11] Lim, K.B., and Giesy, D.P., "Computation of LFT uncertainty bounds with repeated parametric uncertainties," Proceedings of the American Controls Conference, Philadelphia, Pennsylvania, June 24-26, 1998, Paper No. i980106.

[12] Golub, G.H., and Van Loan C.F., Matrix Computations, Johns Hopkins University Press, Baltimore, MD, 1985, pp. 20-24.

[13] Stein, G., and Doyle, J.C., "Beyond singular values and loopshapes," AIAA Journal of Guidance, Control, and Dynamics, Vol. 14, pp. 5-16, 1991.

[14] Optimization Toolbox, The MathWorks, Inc., Natick, MA, 1999. 


\section{List of Tables}

Table 1. Parametric uncertainty and noise allowances.

Table 2. Peak values for $\mu$ and Skewed- $\mu$ for robust performance.

\section{List of Figures}

Figure 1. Uncertainty bound identification framework (top), in canonical form (bottom).

Figure 2. Bode plot of true system (solid), nominal model (dash), and difference (dot) for input $1 /$ output 1.

Figure 3. Smallest additive unmodeled dynamics (o) for model validation, $\bar{\sigma}\left(G_{\text {true }}-\right.$ $\left.G_{n o m}\right)($ dot), fitted weighting functions (solid).

Figure 4. Simulated (true) closed loop frequency response of $\mu$ controllers (top) and Skewed- $\mu$ controllers (bottom). 
Table 1: Parametric uncertainty and noise allowances.

\begin{tabular}{|c|c|c|c|}
\hline & $\bar{\delta}_{1}$ & $\bar{\delta}_{2}$ & $1 / \sqrt{2} * V_{\text {noise }}$ \\
\hline \hline True System & .005 & .01 & \\
\hline Case 1a & .001 & .001 & $\hat{V}_{\text {peak }}$ \\
Case 1b & .001 & .001 & $\hat{V}_{\text {rms }}$ \\
Case 1c & .001 & .001 & $.01 * \hat{V}_{\text {peak }}$ \\
\hline Case 2a & .01 & .01 & $\hat{V}_{\text {peak }}$ \\
Case 2b & .01 & .01 & $\hat{V}_{\text {rms }}$ \\
Case 2c & .01 & .01 & $.01 * \hat{V}_{\text {peak }}$ \\
\hline Case 3a & .05 & .05 & $\hat{V}_{\text {peak }}$ \\
Case 3b & .05 & .05 & $\hat{V}_{\text {rms }}$ \\
Case 3c & .05 & .05 & $.01 * \hat{V}_{\text {peak }}$ \\
\hline
\end{tabular}


Table 2: Peak values for $\mu$ and Skewed- $\mu$ for robust performance.

\begin{tabular}{|c||c|c||c|c|}
\hline Case & $\mu$ controller & $\mu_{\text {peak }}$ & Skewed- $\mu$ controller & Skewed- $\mu_{\text {peak }}$ \\
\hline \hline 1 & $K_{\mu}^{1}$ & 1.57 & $K_{\mu_{s}}^{1}$ & 2.80 \\
2 & $K_{\mu}^{2}$ & 1.49 & $K_{\mu_{s}}^{2}$ & 2.00 \\
3 & $K_{\mu}^{3}$ & 1.35 & $K_{\mu_{s}}^{3}$ & 1.71 \\
\hline \hline
\end{tabular}



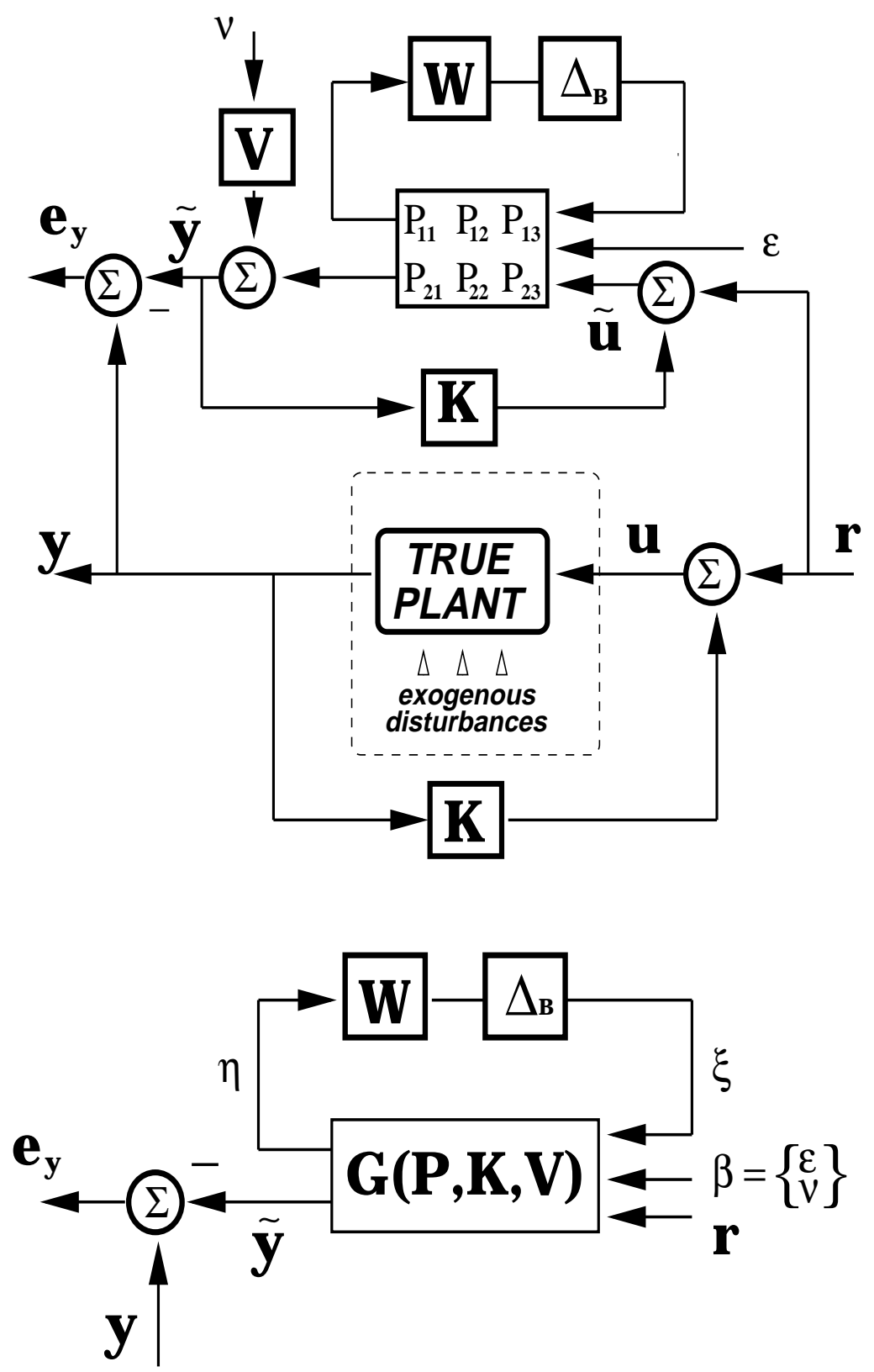

Figure 1: Uncertainty bound identification framework (top), in canonical form (bottom). 

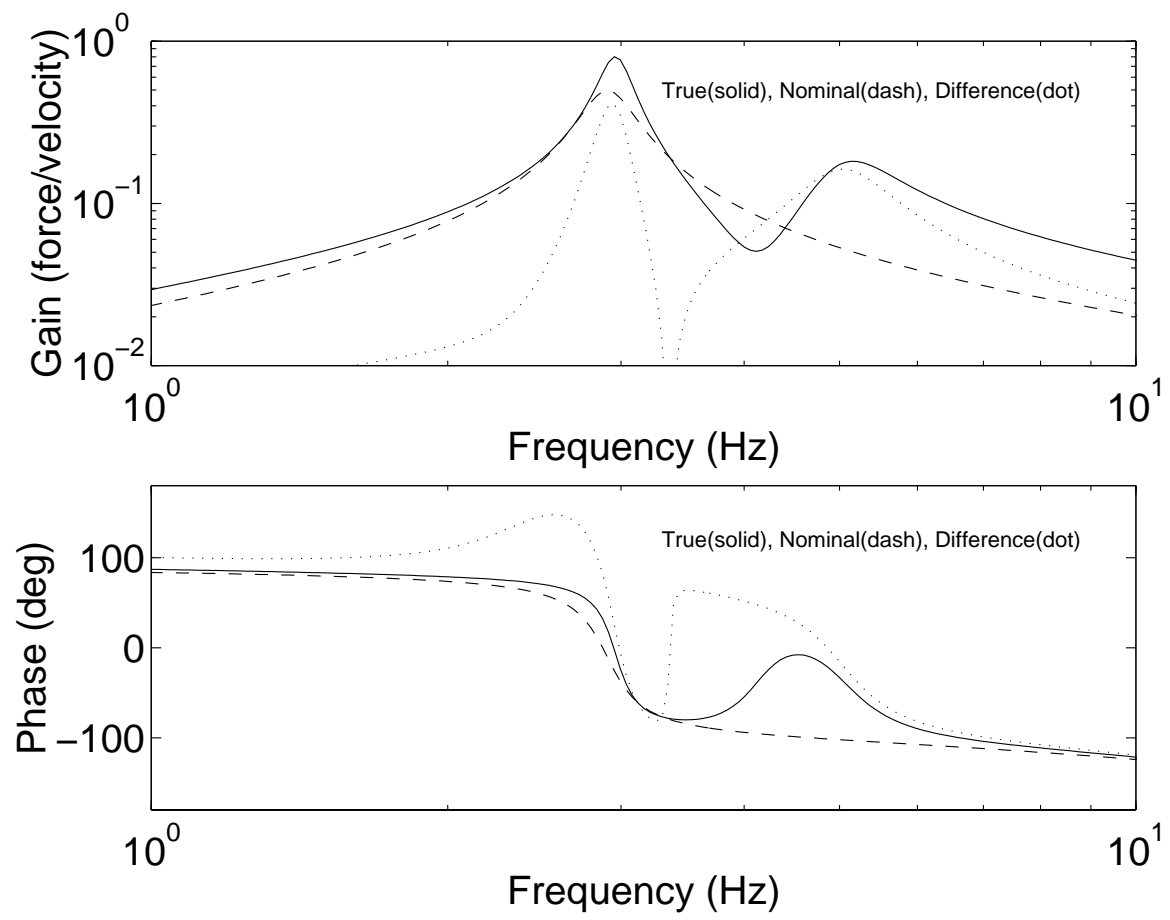

Figure 2: Bode plot of true system (solid), nominal model (dash), and difference (dot) for input 1/output 1 . 

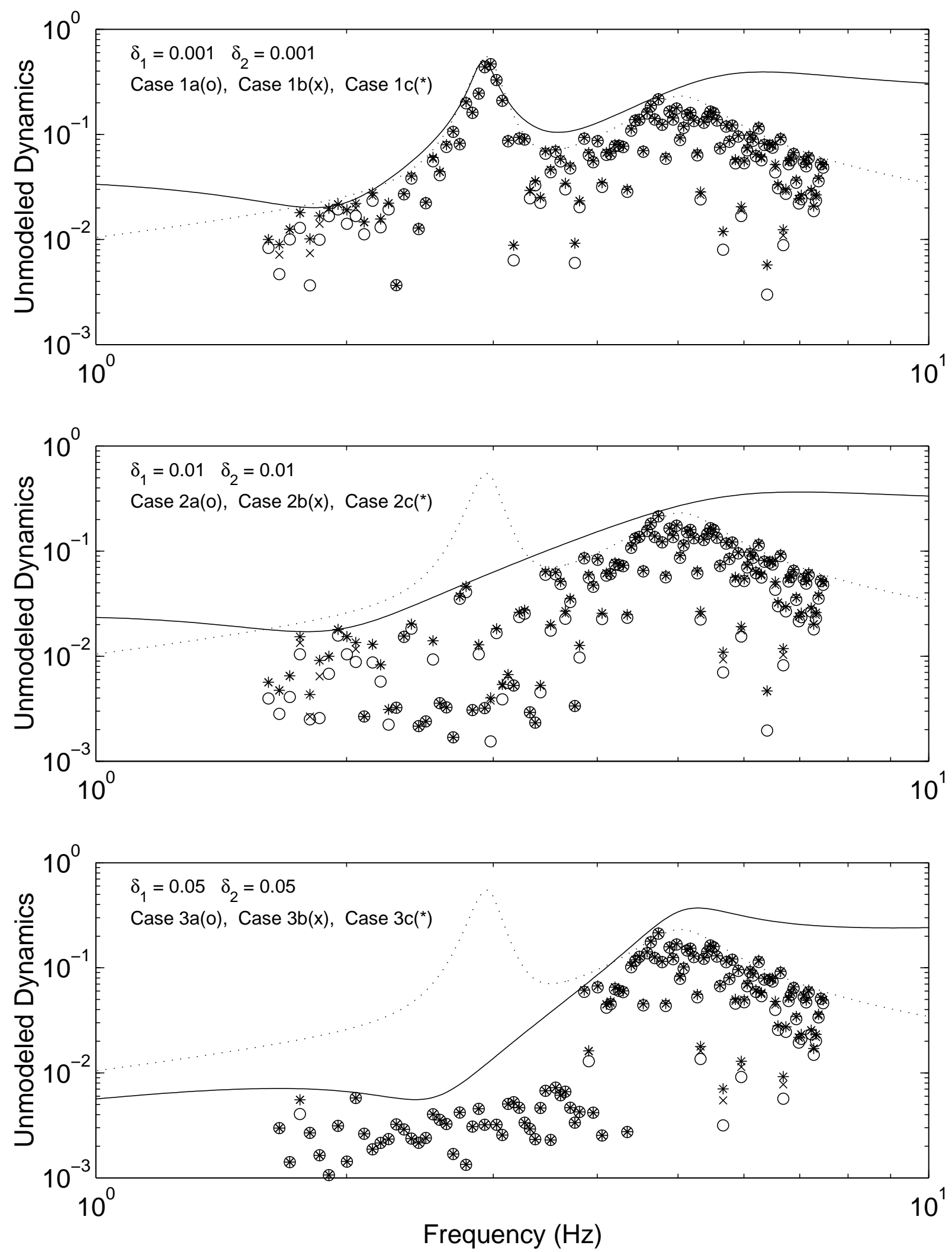

Figure 3: Smallest additive unmodeled dynamics (o) for model validation, $\bar{\sigma}\left(G_{\text {true }}-G_{\text {nom }}\right)$ (dot), fitted weighting functions (solid). 

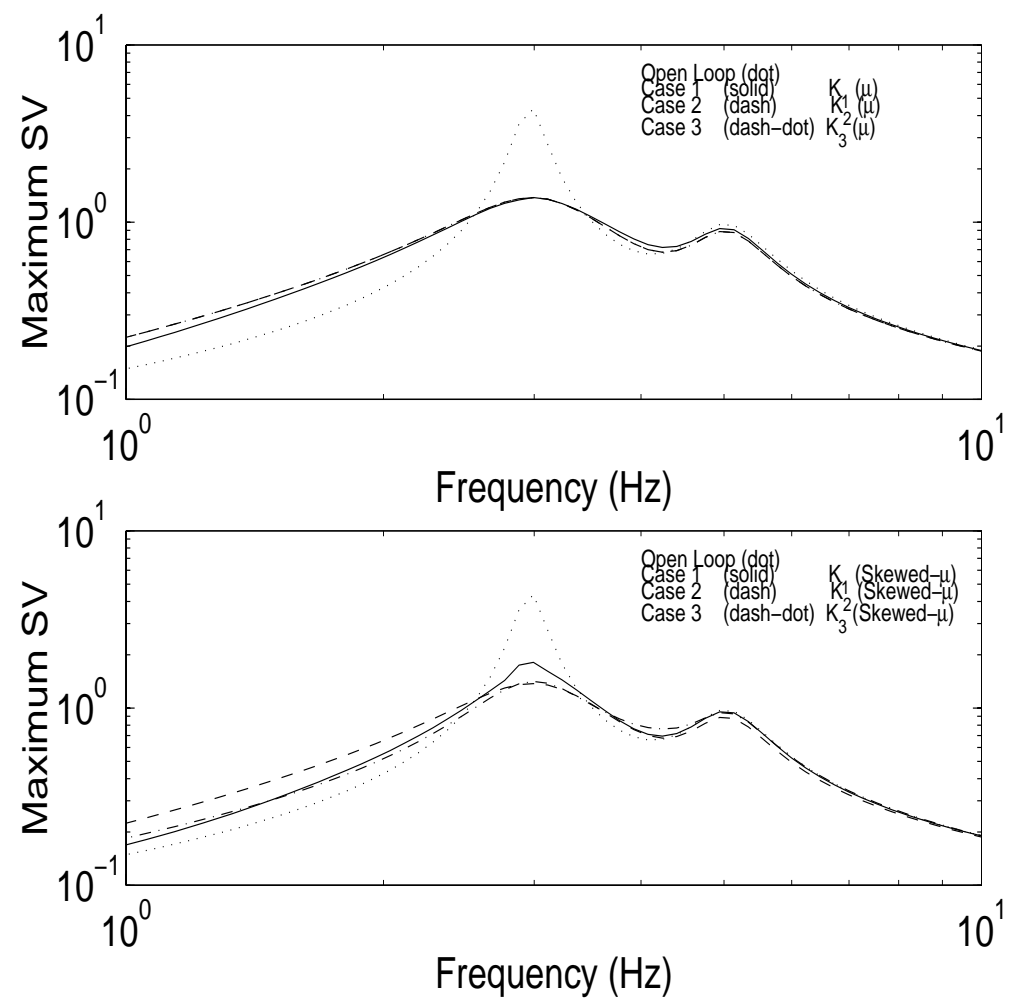

Figure 4: Simulated (true) closed loop frequency response of $\mu$ controllers (top) and Skewed- $\mu$ controllers (bottom). 